\title{
Albuminuria-reducing effect of angiotensin II receptor blocker plus hydrochlorothiazide combination therapy in renal transplant recipients
}

\author{
TOSHIHIDE NAGANUMA, YOSHIAKI TAKEMOTO, JUNJI UCHIDA, TAIYOU OOTOSHI, \\ NOBUYUKI KUWABARA, SATOSHI MAEDA and TATSUYA NAKATANI \\ Department of Urology, Osaka City University Graduate School of Medicine, Osaka, Japan
}

Received February 14, 2012; Accepted March 29, 2012

DOI: $10.3892 / \mathrm{etm} .2012 .542$

\begin{abstract}
In recent years, the combined use of angiotensin II receptor blockers (ARBs) and low-dose diuretics has become clinically possible. Moreover, the GUARD and J-CORE studies have confirmed that the addition of low-dose diuretics to renin-angiotensin system inhibitors reduces albuminuria. In this study, we investigated the clinical effects of a combination drug containing an ARB and a low-dose diuretic in renal transplant recipients. A total of 13 renal transplant recipients who were receiving the maximum dose of the ARB and presenting with microalbuminuria [urine albumin-creatinine ratio (ACR) of 30-300 $\mathrm{mg} / \mathrm{g}$-Cre] were converted to a single pill combination drug containing the same amount of the ARB and $12.5 \mathrm{mg}$ of hydrochlorothiazide (HCTZ) and an intervention study of a crossover trial design was conducted. The clinical parameters were measured at baseline, 3 months after $\mathrm{ARB} / \mathrm{HCTZ}$ conversion and 3 months after reverting to the $\mathrm{ARB}$ and the resulting data were compared. Serum creatinine (S-Cre) and uric acid (UA) levels at 3 months after conversion were significantly higher than those at baseline. The levels of the estimated glomerular filtration rate (eGFR) and ACR at 3 months were significantly lower than those at baseline. S-Cre and UA levels at 3 months after reversion were significantly lower than those at 3 months after conversion. The eGFR and levels of ACR and UA at 3 months after ARB reversion were significantly higher than those at 3 months after conversion. The results of this preliminary study suggest that the combination drug containing an ARB and low-dose diuretic was effective for reducing microalbuminuria in renal transplant recipients. In the future, larger cohort studies are needed to confirm these findings.
\end{abstract}

Correspondence to: Dr Toshihide Naganuma, Department of Urology, Osaka City University Medical School, 1-4-3 Asahi-machi, Abeno-ku, Osaka 545-8585, Japan

E-mail: spxd48k9@aria.ocn.ne.jp

Key words: renal transplant recipients, angiotensin II receptor blockers, hydrochlorothiazide, combination therapy, microalbuminuria

\section{Introduction}

According to clinical practice guidelines for chronic kidney disease (CKD) (1-4), the first choice drugs for hypertension treatment in CKD patients are renin-angiotensin system (RAS) inhibitors and diuretics are mentioned as second choice. In recent years, the combined use of angiotensin II receptor blockers (ARB) and low-dose diuretics, which has been developed to provide maximal antihypertensive effects by using their complementary mechanisms of action, has become clinically possible. Moreover, the GUARD (5) and J-CORE (6) studies have reported that the combination of RAS inhibitors and diuretics reduces albuminuria, indicating that a combination drug with a diuretic may be effective in CKD treatment. This suggests that the combination may also be applied to renal transplant recipients and that it is possible to rescue patients whose blood pressure has not decreased to optimal levels or those who present with albuminuria using conventional treatment. In this study, we investigated the clinical effects of a combination drug containing an ARB and a low-dose diuretic in renal transplant recipients.

\section{Patients and methods}

Study design. An intervention study of a crossover trial design was conducted and the administration protocol was ARB [telmisartan (80 mg) baseline] $\rightarrow$ ARB/hydrochlorothiazide (HCTZ) [telmisartan $(80 \mathrm{mg}) / \mathrm{HCTZ}(12.5 \mathrm{mg})$ single-pill combination drug] $\rightarrow$ ARB [telmisartan $(80 \mathrm{mg})$ ]. The administration period was 3 months for each drug for a total of approximately 6 months. Blood pressure, body weight, albumin-creatinine ratio (ACR), estimated glomerular filtration rate (eGFR), serum creatinine (S-Cre), uric acid (UA), fasting blood sugar, low density lipoprotein, triglyceride, high density lipoprotein, serum potassium and HbA1c levels were measured at baseline, 3 months after ARB/HCTZ conversion and 3 months after reverting to the ARB and the resulting data were compared.

Patients. A total of 134 stable renal transplant recipients who had been followed up at our department for over a year were examined for albuminuria, and microalbuminuria was found 
in a total of 37 patients $(27.6 \%)$. Among them, 13 patients with a S-Cre level of $<2 \mathrm{mg} / \mathrm{dl}$ who had received the maximum dose of the ARB telmisartan for at least 3 months and provided informed consent were converted to the single-pill combination drug with the same dose of telmisartan and $12.5 \mathrm{mg}$ of HCTZ. The discontinuation criteria were allergies or adverse events assessed by the physician-in-charge, surgery or hospitalization and patient withdrawal. During the 6 month period, one patient each withdrew due to surgery at another department, hospitalization for a mental disorder and the onset of gout. As a result, a total of 10 patients completed the protocol and were enrolled in our study. All subjects provided informed consent for participation in the study, which was approved by the human ethics committee of Osaka City University Hospital (Osaka, Japan). The immunosuppression protocols were not changed during the study. The administration pattern of the antihypertensive drugs prior to the study was ARB + angiotensin converting enzyme inhibitor (ACEi) in 5 patients, $\mathrm{ARB}+$ calcium channel blockers $(\mathrm{CCB})$ in 2 patients, $\mathrm{ARB}+\mathrm{ACEi}+\mathrm{CCB}$ in one patient, $\mathrm{ARB}+\mathrm{ACEi}+\alpha$ blocker in one patient and $\mathrm{ARB}+\mathrm{ACEi}+\mathrm{CCB}+\alpha$ blocker in one patient.

Measurements. The subjects were admitted at 8:30 a.m. to our clinical research unit, without having taken immunosuppressive medications, following an 8-12-h overnight fasting period and fasting blood samples were drawn. The low density lipoprotein cholesterol concentration was calculated using the Friedewald formula. Microalbuminuria was defined as a urine ACR of 30 to $300 \mathrm{mg} / \mathrm{g}$-Cre (1). Hypertension was defined as i) the administration of antihypertensive agents and/or a history of this disorder; ii) a systolic blood pressure (SBP) $>140 \mathrm{mmHg}$; or iii) a diastolic blood pressure (DBP) $>90 \mathrm{mmHg}$. The eGFR was calculated using the following equation of the Japanese Society of Nephrology: (194 x age) - (0.287 x serum creatinine) - 1.094 , including a correction factor of 0.739 for women (7).

Statistical analysis. The data are summarized as a percentage or as the mean \pm standard deviation (SD) where appropriate. The changes within each group were evaluated using the paired Student's t-test or Wilcoxon's test. $\mathrm{P}<0.05$ was considered to indicate a statistically significant result. These results were obtained using Stat View V Statistical Software (SAS Institute Inc., Cary, NC, USA).

\section{Results}

Changes in clinical data prior to and following ARB/HCTZ conversion (Table I). S-Cre and UA levels at 3 months after $\mathrm{ARB} / \mathrm{HCTZ}$ conversion were significantly higher than those at baseline. The levels of eGFR and ACR at 3 months after conversion were significantly lower than those at baseline. There was no significant difference in the remaining factors between the values at baseline and 3 months after conversion. S-Cre and UA levels at 3 months after reversion were significantly lower than those at 3 months after conversion. The levels of eGFR, ACR and UA at 3 months after ARB reversion were significantly higher than those at 3 months after conversion. There was no significant difference in the other factors between the values at 3 months after reversion and 3 months after conversion.

Changes in ACR prior to and following ARB/HCTZ conversion (Fig. 1). ACR levels at 3 months after conversion decreased from those at baseline in all patients. ACR levels at 3 months after reversion increased from those at 3 months after conversion in 8 out of 10 patients.

\section{Discussion}

Diuretics are not often administered to renal transplant recipients due to concerns of dehydration and their effects on the metabolic system. However, it was hypothesized that antihypertensive and albuminuria-reducing effects, as shown by the GUARD (5) and J-CORE (6) studies of general populations, may also be expected in renal transplant recipients. In the present study, 10 renal transplant recipients with microalbuminuria uncontrolled with the maximal dose of ARB were converted to a single-pill combination drug containing $12.5 \mathrm{mg}$ of HCTZ and an intervention study of a crossover design was conducted. The ACR and other clinical data were collected at baseline, 3 months after conversion and 3 months after reversion and compared. As a result, ACR and eGFR levels significantly decreased and UA levels significantly increased following the administration of the combination drug. When the patients were reverted to the ARB, these levels returned to the original levels. This is the first study concerning the effects of an ARB/HCTZ combination drug on microalbuminuria in renal transplant recipients.

Microalbuminuria has a well-established association with progressive renal damage (8) and cardiovascular disease (9-11) in general populations. Previous studies in CKD populations have suggested that a decrease in microalbuminuria may lead to the suppression of renal function impairment $(12,13)$ and cardiovascular disease (14-16) progression. In renal transplant recipients, microalbuminuria is a risk factor for graft loss and all-cause mortality (17). The effectiveness of RAS inhibitors for decreasing proteinuria following renal transplantation has been evaluated in various studies $(16,18-20)$. Heinze et al (18) demonstrated that treatment with ACEi and/or ARB led to a 45 and $43 \%$ reduction in the risk of graft loss and patient mortality, respectively. In the present study, the combination drug containing an ARB and low-dose diuretic was found to be effective in decreasing microalbuminuria for patients using the maximal dose of RAS inhibitors. However, as with the GUARD (5) and J-CORE (6) studies, with the decrease in microalbuminuria, there was a marked decrease in eGFR (although the maximum decrease was by $25 \%$ ) in our study. This decrease in eGFR rapidly returned to original levels when the administration of the combination drug was suspended (data not shown). No significant decrease in body weight due to dehydration, which there was concerns for prior to the study, was observed. The use of diuretics is a glomerular preload reduction therapy which reduces eGFR, but evidence concerning the long-term prognosis of renal function has not been established. Further studies concerning the effects of the combination drug on the long-term prognosis of renal function and on cardiovascular disease in renal transplant recipients are required. 
Table I. Changes in clinical data prior to and following ARB/HCTZ conversion.

\begin{tabular}{|c|c|c|c|}
\hline & Baseline & At 3 months after conversion & At 3 months after reversion \\
\hline eGFR $\left(\mathrm{ml} / \mathrm{min} / 1.73 \mathrm{~m}^{2}\right)$ & $47.3 \pm 17.8$ & $41.4 \pm 17.5^{\mathrm{a}}$ & $47.1 \pm 17.9^{c}$ \\
\hline S-Cre (mg/dl) & $1.4 \pm 0.9$ & $1.6 \pm 1.0^{\mathrm{a}}$ & $1.4 \pm 0.8^{\mathrm{b}}$ \\
\hline ACR (mg/g-Cre) & $155.1 \pm 88.8$ & $68.3 \pm 54.9^{\mathrm{a}}$ & $111.1 \pm 80.6^{\mathrm{b}}$ \\
\hline $\mathrm{SBP}(\mathrm{mmHg})$ & $123.6 \pm 13.4$ & $113.6 \pm 7.7$ & $120.7 \pm 9.3$ \\
\hline $\mathrm{DBP}(\mathrm{mmHg})$ & $71.6 \pm 7.1$ & $71.6 \pm 9.7$ & $72.4 \pm 6.2$ \\
\hline Hemoglobin (mg/dl) & $12.9 \pm 2.5$ & $12.7 \pm 2.2$ & $12.8 \pm 2.3$ \\
\hline $\mathrm{BW}(\mathrm{kg})$ & $61.4 \pm 10.7$ & $61.0 \pm 10.9$ & $61.3 \pm 11.2$ \\
\hline $\mathrm{UA}(\mathrm{mg} / \mathrm{dl})$ & $7.1 \pm 1.5$ & $8.3 \pm 1.8^{\mathrm{a}}$ & $7.4 \pm 1.4^{\mathrm{b}}$ \\
\hline $\mathrm{K}(\mathrm{mg} / \mathrm{dl})$ & $4.5 \pm 0.6$ & $4.5 \pm 0.5$ & $4.3 \pm 0.5$ \\
\hline FBS (mg/dl) & $99.7 \pm 11.9$ & $103.2 \pm 22.9$ & $95.8 \pm 18.5$ \\
\hline HbA1c (\%) & $6.1 \pm 1.1$ & $5.8 \pm 1.0$ & $5.5 \pm 1.2$ \\
\hline LDL (mg/dl) & $97.0 \pm 37.2$ & $95.6 \pm 33.8$ & $94.3 \pm 32.1$ \\
\hline TCHO (mg/dl) & $184.2 \pm 48.7$ & $179.0 \pm 44.3$ & $178.8 \pm 45.0$ \\
\hline $\mathrm{TG}(\mathrm{mg} / \mathrm{dl})$ & $112.2 \pm 63.0$ & $131.0 \pm 56.7$ & $117.7 \pm 46.4$ \\
\hline HDL (mg/dl) & $64.8 \pm 21.0$ & $57.2 \pm 11.6$ & $61.0 \pm 16.7$ \\
\hline
\end{tabular}

${ }^{\mathrm{a}} \mathrm{P}<0.01$ vs. value at baseline; ${ }^{\mathrm{b}} \mathrm{P}<0.05$ and ${ }^{\mathrm{c}} \mathrm{P}<0.01$ vs. value at 3 months after conversion. $\mathrm{ARB}$, angiotensin II receptor blocker; HCTZ, hydrochlorothiazide; eGFR, estimated glomerular filtration rate; S-Cre, serum creatinine; ACR, albumin-creatinine ratio; SBP, systolic blood pressure; DBP, diastolic blood pressure; BW, body weight; UA, uric acid; K, potassium; FBS, fasting blood glucose; HbA1c, hemoglobin A1C; LDL, low density lipoprotein; TCHO, high total serum cholesterol; TG, triglycerides; HDL, high density lipoprotein.

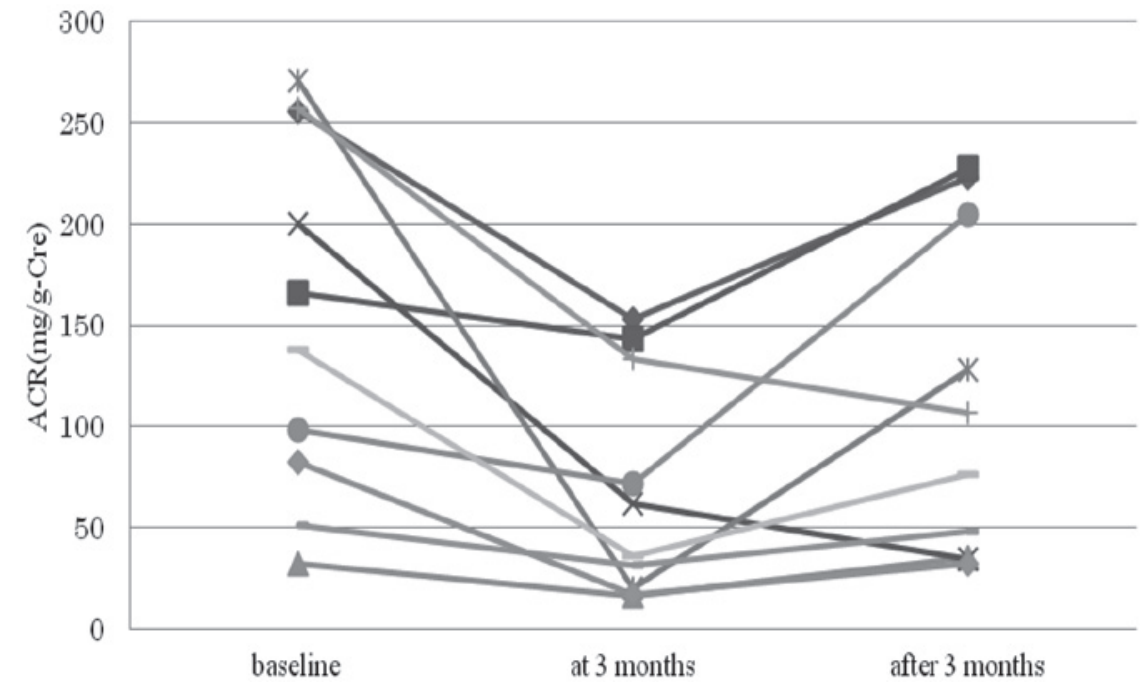

Figure 1. Changes in ACR prior to and following ARB/HCTZ conversion. ACR, albumin-creatinine ratio; ARB, angiotensin II receptor blocker; HCTZ, hydrochlorothiazide.

The diuretic used as the component of the combination drug in this study was a thiazide diuretic and there were concerns about its effects on the metabolic system (21), but its effects on glucose and lipid metabolism were considered to be minimal. However, there was a marked effect on UA metabolism. In the present study, only one dose of the thiazide diuretic HCTZ (12.5 mg) was used, but it may be more appropriate to use a dose of $6.25 \mathrm{mg}$ in order to avoid hyperuricemia. There was hardly any effect on serum potassium levels.

In conclusion, the results of the present study suggest that the ARB/HCTZ combination drug has the potential to be an effective treatment for microalbuminuria, if the decrease in eGFR levels and UA metabolism are carefully monitored. The albuminuria-reducing effect observed in this study may be particularly beneficial for patients receiving the maximal dose of RAS inhibitors. Further studies must be performed concerning indicative cases, drug selection and dosage, long-term renal prognosis and interventional effects on cardiovascular disease. Furthermore, an ARB which is able to improve UA metabolism has been recently developed and its use in combination with HCTZ may bring about beneficial results (22). 


\section{Acknowledgements}

This study was supported by grants from the Osaka City University Medical Research Foundation.

\section{References}

1. National Kidney Foundation: K/DOQI clinical practice guidelines for chronic kidney disease: evaluation, classification, and stratification. Am J Kidney Dis 39 (2 Suppl 1): S1-S266, 2002.

2. Japanese Society of Nephrology: Evidence-based practice guideline for the treatment of CKD. Clin Exp Nephrol 13: 537-566, 2009.

3. Harris D, Thomas M, Johnson D, Nicholls K and Gillin A; Caring for Australasians with Renal Impairment (CARI): The CARI guidelines. Prevention of progression of kidney disease. Nephrology (Carlton) 11 (Suppl 1): S2-S197, 2006.

4. Lamb EJ: United Kingdom guidelines for chronic kidney disease. Scand J Clin Lab Invest Suppl 241: 16-22, 2008.

5. Bakris GL, Toto RD, McCullough PA, Rocha R, Purkayastha D and Davis P: Effects of different ACE inhibitor combinations on albuminuria: results of the GUARD study. Kidney Int 73 1303-1309, 2008.

6. Matsui Y, Eguchi K, O'Rourke MF, Ishikawa J, Shimada K and Kario K: Association between aldosterone induced by antihypertensive medication and arterial stiffness reduction: the J-CORE study. Atherosclerosis 215: 184-188, 2011.

7. Matsuo S, Imai E, Horio M, et al: Revised equations for estimated GFR from serum creatinine in Japan. Am J Kidney Dis 53: 982-992, 2009.

8. Verhave JC, Gansevoort RT, Hillege HL, Bakker SJ, De Zeeuw D and de Jong PE; PREVEND Study Group: An elevated urinary albumin excretion predicts de novo development of renal function impairment in the general population. Kidney Int Suppl: S18-S21, 2004

9. Hillege HL,Fidler V, Diercks GF, et al: Urinary albumin excretion predicts cardiovascular and noncardiovascular mortality in general population. Circulation 106: 1777-1782, 2002.

10. Arnlov J, Evans JC, Meigs JB, et al: Low-grade albuminuria and incidence of cardiovascular disease events in nonhypertensive and nondiabetic individuals: the Framingham Heart Study. Circulation 112: 969-975, 2005.
11. Gerstein HC: Diabetes and the HOPE study: implications for macrovascular and microvascular disease. Int J Clin Pract Suppl: 8-12, 2001.

12. Gaede P, Tarnow L, Vedel P, Parving HH and Pedersen O: Remission to normoalbuminuria during multifactorial treatment preserves kidney function in patients with type 2 diabetes and microalbuminuria. Nephrol Dial Transplant 19: 2784-2788, 2004.

13. Mann JF, Gerstein HC, Yi QL, et al: Progression of renal insufficiency in type 2 diabetes with and without microalbuminuria: results of the Heart Outcomes and Prevention Evaluation (HOPE) randomized study. Am J Kidney Dis 42: 936-942, 2003.

14. Asselbergs FW, Diercks GF, Hillege HL, et al: Effects of fosinopril and pravastatin on cardiovascular events in subjects with microalbuminuria. Circulation 110: 2809-2816, 2004.

15. de Zeeuw D, Remuzzi G, Parving HH, et al: Albuminuria, a therapeutic target for cardiovascular protection in type 2 diabetic patients with nephropathy. Circulation 110: 921-927, 2004.

16. Ibsen $\mathrm{H}$, Olsen $\mathrm{MH}$, Wachtell $\mathrm{K}$, et al: Reduction in albuminuria translates to reduction in cardiovascular events in hypertensive patients: losartan intervention for endpoint reduction in hypertension study. Hypertension 45: 198-202, 2005.

17. Halimi JM, Buchler M, Al-Najjar A, et al: Urinary albumin excretion and the risk of graft loss and death in proteinuric and non-proteinuric renal transplant recipients. Am J Transplant 7: 618-625, 2007.

18. Heinze G, Mitterbauer C, Regele H, et al: Angiotensinconverting enzyme inhibitor or angiotensin II type 1 receptor antagonist therapy is associated with prolonged patient and graft survival after renal transplantation. J Am Soc Nephrol 17: 889-899, 2006.

19. Iñigo P, Campistol JM, Saracho R, et al: Renoprotective effects of losartan in renal transplant recipients. Results of a retrospective study. Nephron Clin Pract 95: c84-c90, 2003.

20. Uchida J, Machida Y, Iwai T, et al: Low-grade albuminuria reduction with angiotensin II type 1 receptor blocker in renal transplant recipients. J Nephrol 24: 515-521, 2011.

21. Palmer BF: Metabolic complications associated with use of diuretics. Semin Nephrol 31: 542-552, 2011.

22. Kita T, Yokota N, Ichiki Y, et al: One-year effectiveness and safety of open-label losartan/hydrochlorothiazide combination therapy in Japanese patients with hypertension uncontrolled with ARBs or ACE inhibitors. Hypertens Res 33: 320-325, 2010 . 